\title{
Factores modificables de riesgo cardiovascular: ¿Cuáles estamos realmente modificando?
}

\author{
Tomás Romero
}

\section{Modifiable cardiovascular risk factors: Which ones are we really modifying?}

While efforts to identify the underlying mechanisms that lead to endovascular atherogenesis continue, the clinical management of the modifiable cardiovascular risk factors should depend on the most advantageous risk-benefit and costeffective therapies. However these efforts probably will not have a significant impact in the population at risk unless an improvement in the socioeconomic factors of cardiovascular risk occurs. The ongoing global obesity epidemic reinforces the need for these changes. Public and privately sponsored programs and policies to improve education and access to health resources are important components of this process (Rev Méd Chile 2009; 137: 1498-501).

(Key words: Atherosclerosis; Coronary artery disease; Obesity)

Recibido el 7 de julio, 2008. Aprobado el 22 de enero, 2009.

Sharp Health Care, San Diego, California, Estados Unidos de Norteamérica.

$\mathrm{E}^{1}$ horizonte de los factores modificables de riesgo cardiovascular, involucrados en el desarrollo de la ateromatosis endovascular, se ha ido expandiendo progresivamente desde 1960. Iniciaron este proceso las contribuciones pioneras del Seven Country Study y el Framingham Heart Study ${ }^{1,2}$ que identificaron el colesterol sanguíneo, la presión arterial, el tabaquismo y la diabetes mellitus como predictores de riesgo cardiovascular. Hoy en día el riesgo de un evento coronario a 10 años puede ser predecido con $75 \%$ de certidumbre usando los datos del estudio de Framingham ${ }^{3}$.

En la última década, factores inflamatorios como la proteína C reactiva, entre otros, que aparecen como nuevos eslabones en la causalidad de la ateromatosis, se han agregado a esta serie de

Correspondencia a: Dr. Tomás Romero, MD. 831 Adella Ave, Coronado (San Diego) CA 92118. USA.

E mail: tomas.romero@sharp.com predictores de riesgo cardiovascular ${ }^{4}$. Más recientemente, los depósitos de calcio en las arterias coronarias han sido mencionados como un factor de riesgo adicional aunque su contribución predictora o causal en relación a los anteriores aún no está bien definida ${ }^{5}$. Probablemente esta lista de factores modificables de riesgo cardiovascular se seguirá extendiendo en el futuro, y los eventos coronarios serán cada vez más predecibles. Sin embargo, para que su identificación en individuos y poblaciones tenga valor desde el punto de vista clínico y de salud pública es necesario esclarecer: 1) qué mecanismos están involucrados en su expresión; 2) cuáles estrategias deben adoptarse para reducir su prevalencia; y 3) en qué medida su corrección reduce el riesgo de eventos cardiovasculares y muerte. En otras palabras, dónde y cómo el riesgo cardiovascular modificable en realidad comienza, se establece y controla.

$\mathrm{Al}$ respecto, es preciso distinguir los factores que actúan como mediadores de aquellos que los antece- 
den favoreciendo su expresión: como ejemplos pueden citarse la hipertensión arterial, trastomos del colesterol, diabetes tipo 2, e incluso marcadores inflamatorios, en relación a la obesidad. Todos éstos, sin embargo, pueden tener a su vez múltiples mediadores adicionales, muchos aún no bien definidos. El esfuerzo de identificar a los así llamados "factores de riesgo independientes", tal como se ha caracterizado a los primeros, tiene sin duda un valor práctico en la predicción individual del riesgo y en especial para decidir tratamientos específicos. No es claro sin embargo cuál ha sido la contribución cuantitativa de éstos en la declinación de la mortalidad cardiovascular que se viene observando desde la década de 1960, cuando esas terapias no estaban aún desamolladas al nivel actual. Un análisis de esta tendencia publicado en 1984 atribuyó a cambios en "el estilo de vida" (disminución del tabaquismo y del nivel del colesterol) $56 \%$ de esta declinación y a terapias específicas sólo 39,5\%, aunque $25 \%$ de ésta fue relacionada a un mejor manejo de la hipertensión arterial $^{6}$. Es importante además notar que la costoefectividad de estas medidas terapéuticas aplicadas a la reducción del niesgo cardiovascular al nivel de poblaciones es menor que la costo-efectividad lograda a través de la mejońa en el estilo de vida, en la que gravitan con más peso los factores que anteceden a los factores de riesgo independientes, como se discutirá más adelante ${ }^{6,7}$.

En relación a la primera pregunta, referente a los mecanismos que generan los factores de riesgo conocidos, la información existente es más bien limitada. Fuera de las raras anomalías monogénicas (genéticas) asociadas a la hipercolesterolemia familiar y algunas formas de hipertensión arterial, todas con un bien definido determinismo mendeliano (mutaciones de genes que incluyen, entre otros, al del receptor del colesterol LDL, de la Apo B-100 y del gene ABCA1, cuya mutación está involucrada en la enfermedad de Tangier, o ausencia de colesterol HDL en el plasma; hipertensión secundaria a la deficiencia de la 11-beta-ketorreductasa del cortisol debido a mutación del gene HSD11B2), existe un creciente campo de investigación, como consecuencia de la rápida expansión de los hallazgos del genoma humano, sobre el determinismo polimórfico o poligenético de la mayoría de los factores de riesgo, identificándose de esta manera múltiples genes posiblemente envueltos en su expresión. Estos actuarían como genes de predis- posición, a diferencia de los primeros que más bien constituyen genes de enfermedad. No parece imposible concebir en el futuro la definición de una especie de "score genético" contribuyendo a la predicción individual de los factores de riesgo modificables tradicionales ${ }^{8-10}$.

Algunos factores claves en el riesgo cardiovascular como la obesidad, tabaquismo y vida sedentaria parecen estar relacionados a lo que ha sido llamado por algunos ${ }^{11,12}$ "causas fundamentales de la enfermedad" y por otros "la causa de las

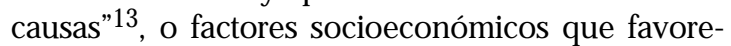
cerían su expresión y prevalencia. Este concepto se remonta en el tiempo a las ideas propuestas por McKweon en la década de $1970^{14}$, quien sostuvo que el incremento de la población mundial y la dramática mejoría de los indicadores de salud de los últimos 200 años se han debido más a los cambios socioeconómicos que a las contribuciones específicas de la ciencia médica o a iniciativas de salud pública en ese período. En contraste, los proponentes de las causas fundamentales plantean que cuando "una sociedad desarrolla los recursos para evitar la enfermedad y la muerte, la habilidad individual para beneficiarse de ellos está determinada por los recursos de conocimiento, dinero, poder, prestigio y conecciones sociales beneficiosas. Quienes los posean ... serán capaces de beneficiarse de los recursos disponibles de salud... a diferencia de quienes carezcan de ellos" ${ }^{\prime 11,12}$.

$\mathrm{Al}$ respecto, estudios realizados en países con tan distinto grado de desarrollo socioeconómico y composición étnica, como Noruega y Chile, han demostrado que mientras más bajo es el nivel educacional y de ingreso, mayor es la prevalencia de obesidad, tabaquismo y de otras conductas nocivas para la salud como el alcoholismo y hábitos sedentarios ${ }^{15-17}$. Sin embargo, no se conocen en definitiva todos los mecanismos a través de los cuales estos factores podrían actuar en el proceso que conduce a la ateromatosis endovascular. Una hipótesis a considerar es que condiciones socioeconómicas desfavorables predispongan a trastornos psicológicos (ansiedad, depresión, autoestima baja, hostilidad), sociales (quiebre del núcleo familiar y de grupo) y a un alto nivel de estrés iniciando de esta manera una cadena causal que mediante la adopción de conductas de salud nocivas (tabaquismo, dieta inapropiada-obesidad, sedentarismo) favorecerían la incidencia de hiper- 
tensión, diabetes tipo II, trastornos de los lípidos. A continuación, un esquema de esta hipótesis:

Factores socioeconómicos desfavorables $\rightarrow$ Efectos psicológicos y sociales (estrés, autoestima baja, depresión, hostilidad, quiebre de núcleo familiar y de grupo) $\rightarrow$ Conductas nocivas para la salud (tabaquismo, dieta inapropiada, sedentarismo, alcoholis$\mathrm{mo}) \rightarrow$ Factores modificables de riesgo cardiovascular (obesidad) $\rightarrow$ Hipertensión arterial, diabetes tipo 2, trastornos de los lípidos) $\rightarrow$ Enfermedad arteriosclerótica cardiovascular.

Pasando a la segunda pregunta, que se refiere a las medidas que podrían reducir la prevalencia de los factores de riesgo conocidos, es necesario volver atrás y revisitar "las causas fundamentales": mejorando el nivel de educación y otros factores socioeconómicos negativos, y por consiguiente apuntando de esta manera a una probable disminución de las conductas nocivas de salud, se podría reducir la prevalencia de obesidad, tabaquismo y sedentarismo y como consecuencia, de la hipertensión y diabetes tipo 2. Aquí no hay ambigüedad en la información que proviene de múltiples experiencias, una de las más importantes, del Framingham Heart Study: en un individuo de 50-60 años portador de un conglomerado de factores que incluya colesterol LDL elevado, tabaquismo, hipertensión y diabetes mellitus, la posibilidad de un evento coronario en un plazo de 10 años es 4 a 6 veces mayor que en la ausencia de ellos ${ }^{3}$. En contraste, la relativamente modesta reducción del riesgo individual $(20 \%$ a $30 \%$ ) que se observa tratando factores de riesgo aislados como la hipertensión y la hipencolesterolemia ${ }^{18,19}$ pone de relieve la importancia de programas y campañas a nivel de poblaciones para intentar un impacto favorable sobre los factores socioeconómicos. En Chile, un estudio reciente de seguimiento de una cohorte de adultos por 8 años ha documentado una relación significativa entre nivel de educación bajo y mortalidad ${ }^{20}$.

La obesidad ha alcanzado globalmente, de acuerdo a un informe reciente de la Organización Mundial de la Salud, proporciones epidémicas y su creciente prevalencia en los niños en muchas regiones del mundo es altamente preocupante por el impacto que a largo plazo tendrá en la incidencia de enfermedades crónicas y cardiovasculares ${ }^{21}$. De persistir esta tendencia, la declinación en la mortalidad cardiovascular que se ha observado en los últimos 60 años podría detenerse o incluso revertir. De acuerdo a este informe, programas destinados a promover hábitos saludables, entre otros, acceso a una alimentación apropiada y oportunidades de actividad física regular son medidas importantes a implementar particularmente en las etapas tempranas de la vida ${ }^{22}$.

Con respecto a la tercera pregunta, múltiples estudios previos han demostrado la reducción de eventos cardiovasculares y muerte tanto en adultos aparentemente libres de expresión clínica de enfermedad coronaria ${ }^{18,19}$ como en individuos después de un episodio isquémico cardiovascular, a través del uso de antilipídicos (especialmente estatinas), inhibidores de la ECA y otras medidas antihipertensivas, aspirina y cesación del tabaquismo, medidas que actúan sobre algunos de los factores modificables ya mencionados de riesgo tradicional ${ }^{23,24}$.

Sin embargo, la comección aislada de algunos de éstos no necesariamente se ha traducido en la reducción del riesgo al que han apuntado ciertos tratamientos. Ejemplos recientes lo constituyen el uso conjunto del torcetrapib y atorvastina (estudio ILUMINATE), que a pesar de producir efectos teónicamente beneficiosos sobre el colesterol (elevación del nivel del HDL en $75 \%$ y reducción del nivel de LDL en $25 \%$ ) aumentó significativamente la mortalidad en comparación al uso aislado de la atorvastina, a pesar de que ésta tuvo efectos mucho más modestos sobre el nivel del colesterol ${ }^{25}$. Otro ejemplo fallido en el enfoque de controlar un marcador metabólico aislado, aunque de reconocida importancia en una enfermedad con alto riesgo cardiovascular como es la diabetes, fue la estrategia de intentar reducir agresivamente el nivel de hemoglobina glicolisada $(\varangle 6 \%)$ en pacientes diabéticos con antecedentes cardiovasculares (estudio ACCORD), lo que resultó en un mayor riesgo de mortalidad comparado al grupo tratado en forma habitual ${ }^{26}$.

En conclusión, mientras los esfuerzos para identificar los mecanismos primarios que originan la ateromatosis endovascular continúan, el manejo clínico del riesgo modificable cardiovascular dependerá de las terapias que aporten el balance más favorable entre riesgo-beneficio y costo-efectividad para combatirlo. Todo este esfuerzo probablemente no tendrá mucho impacto en el riesgo a nivel de la población si no se mejoran las causas fundamentales socioeconómicas del riesgo cardiovascular. La presente epidemia global de obesidad resalta aún más 
la importancia de estos cambios. Programas y políticas educacionales y de acceso a los recursos de

\section{REFERENCIAS}

1. Keys A, Aravanis C, Blackburn HW, Van Buchem FSP, BuZINA R, DJoRDJEvic BS ET AL. Epidemiologic studies related to coronary heart disease: characteristics of men aged 40-59 in seven countries. Acta Med Scand 1967; (Suppl to vol. 460): 1-392.

2. Kagan A, Kannel WB, Dawber TR, Revotskie N. The coronary profile. Ann N YAcad Sci 1963; 97: 883-94.

3. Wilson PW, D'Agostino RB, Levy D, Beianger AM, Silibershatz H, Kannel WB. Prediction of comnary heart disease using risk factor categories. Circulation 1998; 97: 1837-47.

4. Ridker PM, Cushman M, Stampfer MJ, Tracy RP, HeNNEKENS CH. Inflammation, aspirin, and the risk of cardiovascular disease in apparently healthy men. $\mathrm{N}$ Engl J Med 1997; 336: 973-9.

5. Weintraub WS, Diamond GA. Predicting Cardiovascular Events with Coronary Calcium Score. N Engl J Med 2008; 358: 1394-6.

6. Goldman L, Cook EF. The decline in ischemic heart disease mortality rates. An analysis of the comparative effects of medical interventions and changes in lifestyle. Ann Intern Med 1984; 101: 825-36.

7. Urban N. Cost Effectiveness. En: Ockene IS. Ockene JK, ed. Prevention of Coronary Heart Disease. Boston, Toronto, London: Little, Brown and Company, 1992; 555-66.

8. Hobbs HH, Russeil DW, Brown MS, Goldstein JL The LDL receptor locus in familial hypercholesterolemia: mutational analysis of a membrane protein. Annu Rev Genet 1990; 24: 133-70.

9. Q ING WANG. Molecular genetics of coronary artery disease. Curr Opin Cardiol 2005; 20: 182-8.

10. KRISTIANSSON K. Genetics of Cardiovascular Disease: a Candidate Gene Study of USFI. Publications of the National Public Health Institute, A8/2008. (pdf-version) Disponible en: http://www.ktl.fi/portal/4043.

11. LiNK BG, Pheian JC. McKweon and the idea that social conditions are fundamental causes of disease. Am J Public Health 2002; 92: 730-2.

12. LINK BG, PheIAN JC. Understanding sociodemographic differences in health: the role of fundamental social causes. Am J Public Health 1996; 86: 471-3.

13. Мавмот M. The influence of income on health: views of an epidemiologist. Health Affairs 2002; 21: 31-48.

14. Colgrove J. The McKweon thesis: a historical controversy and its enduring influence. Am J Public Health 2002; 92: 725-9.

15. HJermann I, Velive BK, Holme I, Leren P. Effect of diet and smoking intervention on the incidence of coronary heart disease. Report from the Oslo study salud tanto gubernamentales como del sector privado debieran ser parte importante de este proceso.

group of a randomized trial in healthy men. Lancet 1981; 2: 1303-10.

16. Encuesta Nacional de Salud Chile 2003. Pontificia Universidad Católica de Chil, Facultad de Medicina/ Departamento de Salud Pública. Disponible en: http://www.emol.com/noticias/documentos/ informe salud.pdf. Consultado junio 2008.

17. Koch E, Romero T, Manríquez L, Paredes M, Ortúzar E, TAYLOR A ET aL. Desigualdad educacional y socioeconómica como determinante de la mortalidad en Chile: análisis de sobrevida en la cohorte del proyecto San Francisco. Rev Méd Chile 2007; 135: 1370-9.

18. JaMerson K, BaKris GL, DahlöF B et al. Exceptional early blood pressure control rates: the ACCOMPLSH trial. Blood Press 2007; 16: 80-6.

19. Shepherd J, Cobbe SM, Ford I, et aL, for the West of Scotland Coronary Prevention Study Group. Prevention of coronary heart disease with pravastatin in men with hypercholesterolemia. N Engl J Med 1995; 333: 1301-1.

20. Koch E, Romero T, Akel C, Díaz C, Manríquez L, Paredes $M$ ET AL. Impact of cardiovascular risk factors on the relationship between education and mortality. A Chilean prospective cohort study: the San Francisco Project. Circulation 2008; 117: 39-0188 (Abstr.).

21. Word Heatth Organization 2008 Report. Contacts: Puska P, Nishida C, Porter D. Global Strategy on Diet, Physical Activity and Health. Obesity and Overweight. Disponible en: http://www.who.int/dietphysicalactivity/media/ en/gsfs_obesity.pdf. Consultado en junio 2008.

22. Pearson ta, Blair R, Daniels SR, Eckel RH, Ekel RH, FAIR JM ET AL. AHA Guidelines for Primary Prevention of Cardiovascular Disease and Stroke: 2002 Update: Consensus Panel Guide to Comprehensive Risk Reduction for Adult Patients Without Coronary or Other Atherosclerotic Vascular Diseases. Circulation 2002; 106 388-91.

23. Fonarow GC, Gawlinski A, Moughrabi S, Tiwish JH. Improved treatment of coronary heart disease by implementation of a Cardiac Hospitalization Atherosclerosis Management Program (CHAMP) Am J Cardiol 2001; 87: 819-22.

24. ROMERo T. La rehabilitación cardíaca como primer paso en la Prevención Secundaria. Rev Méd Chile 2000; 128: 787-98.

25. NisSEN SE, TARDIF JC, Nichols SJ ET aL. Effects of torcetrapib on the progression of coronary atherosclerosis. N Enl J Med 2007; 356: 1304-16.

26. The Action to Control Cardiovascular Risk in Diabetes Study Group (ACCORD). Effects of intensive glucose lowering in Type 2 diabetes. N Engl J Med 2008; 358: 2545-59. 